\title{
Efficacy of switching to dolutegravir plus rilpivirine, the small-tablet regimen, in patients with dysphagia: two case reports
}

Takefumi Suzuki ${ }^{1}$, Nobuko Hara', Morichika Osa ${ }^{2}$, Kazuhisa Misawa², Kazuo Imai ${ }^{2}$, Yuji Fujikura², Takuya Maeda ${ }^{2,45^{*}}$ (D), Wataru Sonehara ${ }^{1,3}$ and Akihiko Kawana ${ }^{2}$

\begin{abstract}
Background: The advent of well-tolerated and effective anti-retroviral drugs against human immunodeficiency virus-1 (HIV-1) infection has been a major step forward that has achieved long-term survival in recent years. The number of HIV-1 infected patients who experience difficulty in swallowing tablets is expected to increase as the HIV-infected population advances in age or develops comorbidities or treatment sequelae affecting the central nervous system.
\end{abstract}

Case presentation: Here, we describe two HIV-1-infected patients who experienced progressive dysphagia leading to inability to swallow the antiretroviral tablets included in the standard regimen. Both patients had a plasma viral load $<40$ copies $/ \mathrm{mL}$ while receiving anti-retroviral therapy with the recommended combination antiretroviral therapy (CART) regimen, but the dysphagia necessitated a switch. By switching to much smaller sized combined regimen of dolutegravir (DTG) plus rilpivirine (RPV) tablets, both of our patients were able to successfully continue treatment and maintain adherence without the need for crushing tablets or preparing an oral suspension. Additionally, switching from the recommended CART regimen to DTG plus RPV successfully maintained viral suppression. At the last available follow-up (12 months after switching to DTG/RPV), HIV-1 viral load remained below the lower limit of quantification.

Conclusions: An alternative therapeutic option that takes tablet size into consideration could not only contribute to improved patient adherence, but also a reduced care burden for HIV-infected patients with dysphagia. Thus, switching to the "small-tablet regimen" of DTG plus RPV has the potential to improve the survival and well-being of patients with dysphagia.

Keywords: Dolutegravir, Rilpivirine, Dysphagia, NRTI-sparing

\section{Background}

The advent of well-tolerated and effective anti-retroviral drugs against human immunodeficiency virus-1 (HIV-1) infection has been a major step forward that has achieved long-term survival in recent years [1]. Moreover, the number of HIV-1 infected patients who experience difficulty in swallowing tablets is expected to

\footnotetext{
* Correspondence: t_maeda@saitama-med.ac.jp

${ }^{2}$ Division of Infectious Diseases and Pulmonary Medicine, Department of Internal Medicine, National Defense Medical College, 3-2, Namiki, Tokorozawa-shi, Saitama 359-8513, Japan

${ }^{4}$ Department of Microbiology, Saitama Medical University, 38 Morohongo,

Moroyama-Machi, Iruma-Gun, Saitama 350-0495, Japan

Full list of author information is available at the end of the article
}

increase as the HIV-infected population advances in age or develops comorbidities or treatment sequelae affecting the central nervous system [2]. With the long lifespan conferred by combination antiretroviral therapy (cART), the patient population affected by HIV-associated dementia (HAND) or Alzheimer's disease is also growing. The standard regimens of cART consist of two nucleoside/ nucleotide reverse transcriptase inhibitors (NRTIs) plus a third drug, so it would be beneficial to reduce both the number of drugs and the tablet size for the medication to be easy to take and have good tolerability for all patients over the long term $[1,3]$. 
The Food and Drug Administration (FDA) has previously suggested that patient adherence to medication regimens can be influenced by the size and shape of a tablet or capsule, and size specifically as the main reason for the difficulty in swallowing [4]. NRTI combination drugs (e.g., tenofovir/emtricitabine; TDF/FTC, abacavir/ lamivudine; $\mathrm{ABC} / 3 \mathrm{Tc}$ ), including all recommended and alternative regimens in 2015, are $>17 \mathrm{~mm}$ at their largest dimension and, despite being oval-shaped, can be difficult to swallow. Conversely, liquid formulations such as Kaletra ${ }^{\circledR}$ suspension are easier to administer, but caregivers would have the burden of refrigerating and measuring the liquids each day. Furthermore, Kaletra contains a high concentration of alcohol and therefore has the potential to lead to significant alcohol toxicity. Unfortunately, accepted and sufficiently safe liquid formulations are not yet licensed in Japan.

Dolutegravir (DTG) is a next-generation anti-retroviral drug, an integrase inhibitor with a long intracellular halflife that allows once daily dosing without the need for any boosting drugs $[5,6]$. Its major metabolic pathway involves uridine diphosphate glucuronosyltransferase-1A1 with a minor metabolic component of cytochrome P450 isoforms, and therefore its interactions with co-medications are quite limited [7]. It is also noteworthy that its tablet size $(9.1 \mathrm{~mm})$, along with that of rilpivirine (RPV) which is the smallest tablet $(6.4 \mathrm{~mm})$ among non-NRTIs, is small enough for most patients to take orally. In recent years, several studies have focused on DTG monotherapy or DTG-based lightened regimens because of its powerful anti-viral effect, ease of medication, and excellent tolerability $[8,9]$. Although the clinical effectiveness and safety of the combined regimen with DTG plus RPV is controversial and remains to be established, it has the potential to enable continued successful treatment and maintain adherence without the need for crushing or preparing an oral suspension when administering the drug to older patients or patients with dysphagia.

We describe here two cases of HIV-1-infected patients whose comorbidities involving the central nervous system (CNS) and/or aging led to difficulty swallowing the anti-retroviral tablets. Switching from the recommended cART regimen to a "small-tablet regimen" of DTG plus RPV, after patients consented to the unestablished regimen with the aim of treating their disease, successfully maintained viral suppression.

\section{Case presentation \\ Case 1}

The patient was a Japanese man with HIV-1 infection who also had multiple system atrophy (MSA), a progressive neurodegenerative disorder characterized by cerebellar ataxia, parkinsonism, and autonomic dysfunction. At age 59 years, he presented with complaints of decreased vision and myodesopsia in both eyes. The uveitis workup revealed positive serology for syphilis and HIV-1 with a CD4 cell count of 354 cells/ $\mu \mathrm{L}$ and a HIV-1 viral load of $8.3 \times 10^{3}$ copies $/ \mathrm{mL}$. At age 61 , he was diagnosed with left pulmonary adenocarcinoma of pathological stage pT3N0M0, pStage IIB and underwent left upper lobectomy and irradiation with 60 Gy (total dose). Prior to this surgery, anti-retroviral therapy was initiated with a cART regimen of raltegravir (RAL) plus TDF/FTC according to the national protocol. Although the HIV-1 viral load became undetectable during the first 12 months of therapy, he was switched to RAL plus ABC/3Tc at age 62 because he subsequently presented with reduced renal function as a side effect of the original therapy. Thereafter, he experienced asymptomatic orthostatic hypotension, slowed movement, progressive gait instability, and severe constipation. All these symptoms and signs persisted without cognitive changes, and co-treatment with levodopa/ carbidopa and a dopamine agonist was started. At a medical evaluation at age 65 years in our hospital, brain magnetic resonance imaging (MRI) showed the "hot cross bun" sign in the pons, as well as cerebellar and brainstem atrophy [10]. Finally, MSA was diagnosed and taltirelin hydrate was added to his treatment. However, symptom progression continued, and additional progressive symptoms of dysphagia became apparent; therefore, oral tablets of levodopa/carbidopa, a dopamine agonist, and taltirelin hydrate had to be provided after crushing.

At age 66, he was switched to DTG/RPV dual therapy because the original anti-retroviral drug tablets were too large for him to take orally. At the last available followup (12 months after switching to DTG/RPV), HIV-1 viral load remained below the lower limit of quantification (<40 copies/mL) and CD4 cell count was maintained at 474 cells/mL (Fig. 1). Although the dysphagia persisted and progressed, this change to a "small-tablet regimen" reduced the burden on his caregivers at home and enabled the patient to take the tablets on his own without needing to crush them for oral suspension.

\section{Case 2}

The patient was a Japanese woman with HIV-1 infection and neurologic sequelae with progressive multifocal leukoencephalopathy (PML) showing fatal subacute demyelinating disease of the brain that occurs in immunosuppressed patients. At age 30 years, she presented to our hospital with complaints of progressive confusion, severe dyspnea, and asthenia. Blood testing showed a positive serology for HIV-1 with a CD4 cell count of 116 cells $/ \mu \mathrm{L}$ and a HIV-1 viral load of $7.2 \times 10^{3}$ copies $/ \mathrm{mL}$. She underwent an extensive examination and based on clinical and brain MRI findings was finally diagnosed with pneumocystis pneumonia (PCP) complicated with possible PML. Despite attempts to optimize gas exchange and 


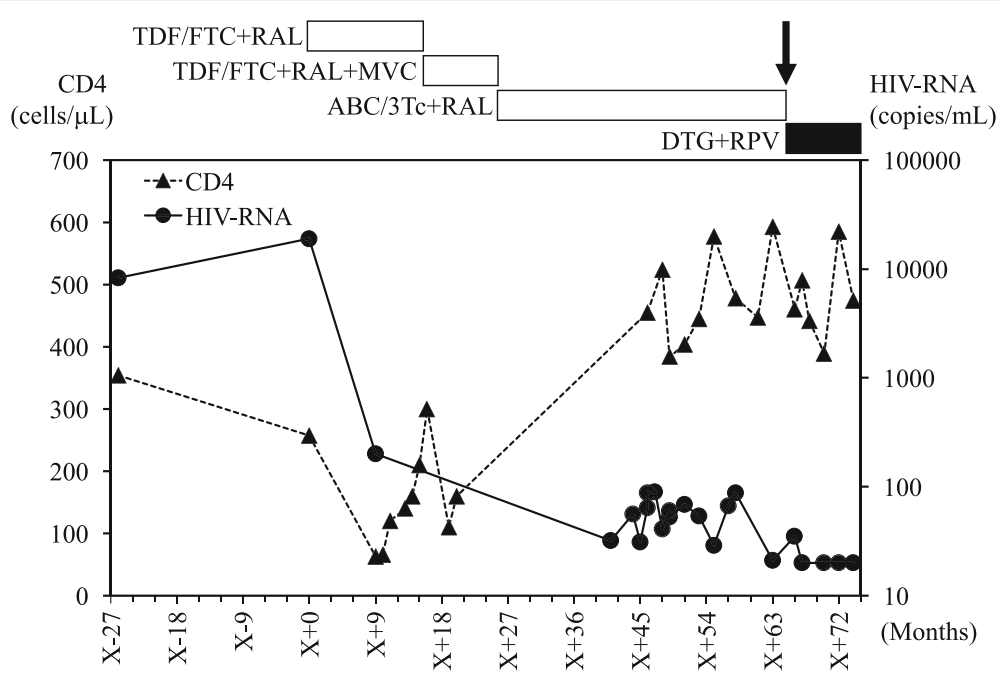

Fig. 1 Plasma viral load and CD4 cell count follow-up from 2008 to 2016 in Case 1. Arrow indicates the time of switching to DTG/RPV dual therapy. $X+0$ indicates the time of first administration of anti-retroviral drugs. Abbreviations: TDF/FTC, tenofovir/emtricitabine; ABC/3Tc, abacavir/lamivudine; RPV, rilpivirine; DRV/r, darunavir/ritonavir; MVC, maraviroc; RAL, raltegravir; DTG, dolutegravir

control infection with various antimicrobial agents, she required intubation and mechanical ventilation for a long duration. Anti-retroviral therapy was introduced via the feeding tubes primarily with crushed tablets of lopinavir/ ritonavir (LPV/r) plus TDF/FTC. Consequently, HIV-1 viral load decreased and became consistently undetectable. She was also administered enteral nutrition via the same feeding tube. At age 32, she was switched to cART with $\mathrm{EFV}$ plus $\mathrm{ABC} / 3 \mathrm{Tc}$ because she presented with pathological bone fractures and demineralization. Alongside intensive anti-retroviral treatment, simultaneous dysphagia and respiratory rehabilitation for more than 3 years led to progressive improvement of in her symptoms [11]. At age 36, she was successfully extubated without respiratory distress and was able to resume oral feeding. However, because the original anti-retroviral drug tablets were too large for her to take orally, she was switched to DTG/RPV dual therapy.

At the last available follow-up (12 months after switching to DTG/RPV), HIV-1 viral load remained below the lower limit of quantification $(<40$ copies $/ \mathrm{mL})$ and $\mathrm{CD} 4$ cell count was maintained at 289 cells/mL (Fig. 2).

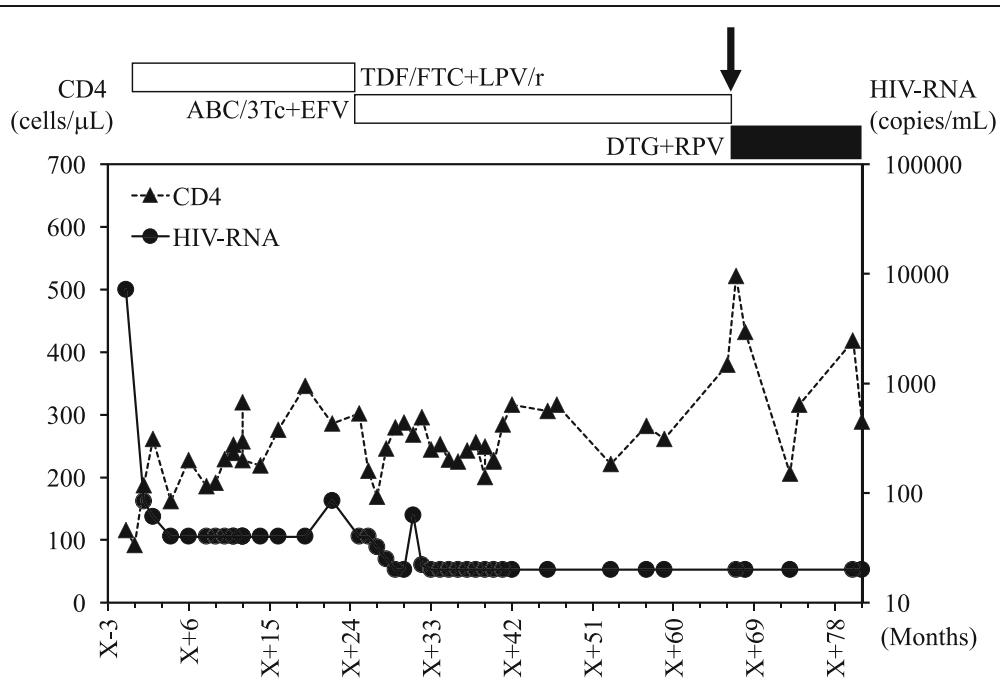

Fig. 2 Plasma viral load and CD4 cell count follow-up from 2009 to 2016 in Case 2. Arrow indicates the time of switching to DTG/RPV dual therapy. $X+0$ indicates the time of first administration of anti-retroviral drugs. Abbreviations: TDF/FTC, tenofovir/emtricitabine; ABC/3Tc, abacavir/lamivudine; RPV, rilpivirine; LPV/r, lopinavir/ritonavir; EFV, efavirenz; DTG, dolutegravir 


\section{Discussion}

The CNS is a major site of disease in HIV-infected patients because it serves as a reservoir for the virus as well as a site for opportunistic infections [12]. Indeed, just short of $40 \%$ of HIV-infected patients will have some neuromuscular manifestations such as dysphagia. Moreover, due to the significantly reduced mortality of these patients, the impact of the aging combined with their neuromuscular issues accelerate their need for care [13]. Previously, anti-retroviral treatment itself led to side effects such as dysphagia and various gastric symptoms including nausea, vomiting, gastroesophageal reflux, and taste aversions. However, with drastic advances in cART, recent treatment strategies have led to fewer side effects, increased efficacy, and higher virologic response in clinical practice. Therefore, adherence to cART has become one of the major independent predictors of long-term treatment success in HIV patients.

In past studies, if the diameter of the tablet exceeded $8 \mathrm{~mm}$, there was a high possibility that oral administration could become difficulty for adult patients. In addition, increasing tablet or capsule size is also believed to correlate with increasing difficulty with oropharyngeal transfer [14, 15]. Therefore, treatment constituting a combination of tablets, is suitable and therefore can be reasonably defined as a "small-tablet-regimen". By switching to the much smaller tablets of the combined regimen of DTG $(9.1 \mathrm{~mm})$ plus RPV $(6.4 \mathrm{~mm})$ tablets once a day at home, both of our patients were able to continue successful treatment and maintain adherence without the need for crushing or preparing an oral suspension. Additionally, the burden on caregivers of maintaining the oral administration of the drugs was reduced compared with administering the medications via feeding tubes, and the efficacy of the drug and the stability of the preparation were also ensured [16]. Thus, switching to the "small-tablet regimen" of DTG plus RPV has the potential to improve the survival and well-being of patients with dysphagia.

The NRTI class (e.g., ABC, TDF) remains a key component of most antiretroviral regimens used in current HIV clinical practice. However, there are clinical situations in which eliminating NRTI exposure is desirable (e.g., in patients with a high risk of cardiovascular disease [17], positive HLA-B*5701 [18, 19], chronic kidney disease [20-22], or osteoporosis [23]). As noted in the 2016 guidelines from the US Department of Health and Human Services, two NRTI-sparing regimens-darunavir $(\mathrm{DRV}) / \mathrm{r}$ plus RAL and LPV/r plus $3 \mathrm{Tc}$ - should be considered as alternative regimens when $\mathrm{ABC}$ or TDF cannot be used [3].

NEAT001/ANRS143, a fully powered trial, demonstrated that DRV/r plus RAL as first-line anti-retroviral therapy was non-inferior to the standard treatment and thus presented a therapeutic option for patients with baseline CD4 cell counts $>200 / \mu \mathrm{L}$ [24]. A small singlearm study of DRV/r plus RAL, however, showed high rates of virologic failure in patients with baseline viral load >100,000 copies/mL [25]. In the GARDEL study, the $\mathrm{LPV} / \mathrm{r}$ plus 3Tc regimen was better tolerated than the $\mathrm{LPV} / \mathrm{r}$ plus 2-NRTI regimen, although $\mathrm{LPV} / \mathrm{r}$ is not considered a viable alternative because of its metabolic complications and pill burden [26]. Together, these data suggest that NRTI-sparing regimens have weaker efficacy than the recommended regimens. On the other hand, it has been reported that the NRTI-sparing regimen was efficacious and safe as a replacement regimen especially in patients showing long-lasting virologic suppression. A particularly attractive option currently under study is the long-acting combination of an integrase inhibitor plus a non-NRTI, the most metabolism-friendly antiretroviral drug class, for the maintenance of viral suppression [27]. The SWORD-1 and SWORD-2 sponsor-initiative clinical trials are currently ongoing to assess the antiviral activity and safety of DTG plus RPV and a current antiretroviral therapy for 48 weeks in patients with suppressed viral load [28].

With the rapid increase in the number of elderly HIVinfected patients worldwide, comorbidities associated with aging, such as dysphagia, are expected to become significant factors affecting treatment outcomes and patient quality of life. An alternative therapeutic option that takes tablet size into consideration could not only contribute to improved patient adherence, but also a reduced care burden for HIV-infected patients with dysphagia. Further studies are needed to confirm the long-term efficacy and safety of the DTG plus RPV regimen in HIV-infected patients with suppressed viral load.

\section{Conclusions}

DTG + RPV dual therapy is effective in patients with difficulty swallowing, can be considered as alternative regimens for the maintenance of viral suppression.

\section{Abbreviations \\ CART: Combination antiretroviral therapy; DTG: Dolutegravir; NRTI: Nucleoside reverse transcriptase inhibitors; PI: Protease inhibitors; RPV: Rilpivirine}

\section{Acknowledgements}

Not applicable.

\section{Funding}

The authors received no financial support for the research, authorship, and/or publication of this article.

\section{Availability of data and materials}

Data used in this case report will not be shared due to the risk of identifying an individual, although most patient's data are presented in the main paper.

\section{Authors' contributions}

TS and TM wrote the manuscript. NH, MO, KM, Kl, YF, WS, and AK helped to draft the manuscript. All authors read and approved the final manuscript. 


\section{Ethics approval and consent to participate}

This study was approved by the National Defense Medical College Research Ethics Committee (reference 2270). Written informed consent was obtained from the patients for this case report.

\section{Consent for publication}

A copy of the written consent is available for review by the Editor-in-Chief of this journal.

\section{Competing interests}

The authors declare that they have no competing interest.

\section{Publisher's Note}

Springer Nature remains neutral with regard to jurisdictional claims in published maps and institutional affiliations.

\section{Author details}

'Department of Pharmacy, National Defense Medical College Hospital, 3-2, Namiki, Tokorozawa-shi, Saitama 359-8513, Japan. ${ }^{2}$ Division of Infectious Diseases and Pulmonary Medicine, Department of Internal Medicine, National Defense Medical College, 3-2, Namiki, Tokorozawa-shi, Saitama 359-8513, Japan. ${ }^{3}$ Department of Pharmacy, Mishuku Hospital, 5-33-12, Kamimeguro, Meguro-ku, Tokyo 153-0051, Japan. ${ }^{4}$ Department of Microbiology, Saitama Medical University, 38 Morohongo, Moroyama-Machi, Iruma-Gun, Saitama 350-0495, Japan. ${ }^{5}$ Center for Clinical Infectious Diseases and Research, Saitama Medical University, 38 Morohongo, Moroyama-Machi, Iruma-Gun, Saitama 350-0495, Japan.

Received: 31 May 2017 Accepted: 11 September 2017

Published online: 19 September 2017

\section{References}

1. Günthard HF, Aberg JA, Eron JJ, Hoy JF, Telenti A, Benson CA, et al. Antiretroviral treatment of adult HIV infection: 2014 recommendations of the international antiviral society-USA panel. JAMA. 2014;312:410-5.

2. Work Group for HIV Aging Consensus Project. Summary report from the human immunodeficiency virus and aging consensus project: treatment strategies for clinicians managing older individuals with the human immunodeficiency virus. J Am Geriatr Soc. 2012;60:974-9.

3. DHHS, US Department of Health and Human Services (DHHS). Panel on antiretroviral guidelines for adults and adolescents. Guidelines for the Use of Antiretroviral Agents in HIV-1-Infected Adults and Adolescents. $<$ https:/aidsinfo.nih.gov/contentfiles/lvguidelines/adultandadolescentgl.pdf>

4. U.S. Department of Health and Human Services, Food and Drug Administration. Size, shape, and other physical attributes of generic tablets and capsules guidance for industry. June 2015

5. Greig SL, Deeks ED. Abacavir/dolutegravir/lamivudine single-tablet regimen: a review of its use in HIV-1 infection. Drugs. 2015;75:503-14.

6. Min S, Sloan L, De Jesus E, Hawkins T, McCurdy L, Song I, et al. Antiviral activity, safety, and pharmacokinetics/pharmacodynamics of dolutegravir as 10-day monotherapy in HIV-1-infected adults. AIDS. 2011;25:1737-45.

7. Cottrell ML, Hadzic T, Kashuba AD. Clinical pharmacokinetic, pharmacodynamic and drug-interaction profile of the integrase inhibitor dolutegravir. Clin Pharmacokinet. 2013;52:981-94.

8. Gubavu C, Prazuck T, Niang M, Buret J, Mille C, Guinard J, et al. Dolutegravir-based monotherapy or dual therapy maintains a high proportion of viral suppression even in highly experienced HIV-1-infected patients. J Antimicrob Chemother. 2016;71:1046-50

9. Rojas J, Blanco JL, Marcos MA, Lonca M, Tricas A, Moreno L, et al. Dolutegravir monotherapy in HIV-infected patients with sustained viral suppression. J Antimicrob Chemother. 2016;71:1975-81.

10. Deguchi K, Ikeda K, Kume K, Takata T, Kokudo Y, Kamada M, et al. Significance of the hot-cross bun sign on T2*-weighted MRI for the diagnosis of multiple system atrophy. J Neurol. 2015;262:1433-9.

11. Jamroz-Wiśniewska A, Jaworski J, Suszek D, Janczarek M, Stelmasiak Z, Rejdak K, et al. Disappearance of white matter lesions on MRI and clinical recovery after initiating antiretroviral therapy in a case of HIV infection presenting as spastic paraparesis. Neurol Neurochir Pol. 2014;48:378-81.

12. Brew BJ. Neurological Sequelae of primary HIV infection. Handb Clin Neurol. 2007;85:69-77.

13. Mochan A, Modi M, Modi G. Stroke in black south African HIV-positive patients: a prospective analysis. Stroke. 2003;34:10-5.
14. Hey H, Jørgensen F, Sørensen K, Hasselbalch H, Wamberg T. Oesophageal transit of six commonly used tablets and capsules. Br Med J (Clin Res Ed). 1982;285:1717-9.

15. Channer KS, Virjee JP. The effect of size and shape of tablets on their esophageal transit. J Clin Pharmacol. 1986;26:141-6.

16. Jackson LD, Little J, Kung E, Williams EM, Siemiatkowska K, Plowman S. Safe medication swallowing in Dysphagia: a collaborative improvement project. Healthc Q. 2008;11:110-6.

17. Cruciani M, Zanichelli V, Serpelloni G, Bosco O, Malena M, Mazzi R, et al. Abacavir use and cardiovascular disease events: a meta-analysis of published and unpublished data. AIDS. 2011;25:1993-2004.

18. Mallal S, Phillips E, Carosi G, Molina JM, Workman C, Tomazic J, et al HLA-B*5701 screening for hypersensitivity to abacavir. N Engl J Med. 2008;358:568-79.

19. Saag M, Balu R, Phillips E, Brachman P, Martorell C, Burman W, et al. High sensitivity of human leukocyte antigen-b*5701 as a marker for immunologically confirmed abacavir hypersensitivity in white and black patients. Clin Infect Dis. 2008;46:1111-8.

20. Nishijima T, Kawasaki Y, Tanaka N, Mizushima D, Aoki T, Watanabe K, et al. Long-term exposure to tenofovir continuously decrease renal function in HIV-1-infected patients with low body weight: results from 10 years of follow-up. AIDS. 2014;28:1903-10.

21. Goicoechea M, Liu S, Best B, Sun S, Jain S, Kemper C, et al. Greater tenofovir-associated renal function decline with protease inhibitor-based versus nonnucleoside reverse-transcriptase inhibitor-based therapy. J Infect Dis. 2008;197:102-8.

22. Gallant JE, Moore RD. Renal function with use of a tenofovir-containing initial antiretroviral regimen. AIDS. 2009;23:1971-5.

23. Reynes J, Trinh R, Pulido F, Soto-Malave R, Gathe J, Qaqish R, et al. Lopinavir/ritonavir combined with raltegravir or tenofovir/emtricitabine in antiretroviral-naive subjects: 96-week results of the PROGRESS study. AIDS Res Hum Retrovir. 2013;29:256-65.

24. Raffi F, Babiker AG, Richert L, Molina JM, George EC, Antinori A, et al. Ritonavir-boosted darunavir combined with raltegravir or tenofoviremtricitabine in antiretroviral-naive adults infected with HIV-1: 96 week results from the NEAT001/ANRS143 randomised non-inferiority trial. Lancet. 2014:384:1942-51.

25. Taiwo B, Zheng L, Gallien S, Matining RM, Kuritzkes DR, Wilson CC, et al. Efficacy of a nucleoside-sparing regimen of darunavir/ritonavir plus raltegravir in treatment-naive HIV-1-infected patients (ACTG A5262). AIDS. 2011;25:2113-22.

26. Cahn P, Andrade-Villanueva J, Arribas JR, Gatell JM, Lama JR, Norton M, et al. Dual therapy with lopinavir and ritonavir plus lamivudine versus triple therapy with lopinavir and ritonavir plus two nucleoside reverse transcriptase inhibitors in antiretroviral-therapy-naive adults with HIV-1 infection: 48 week results of the randomised, open label, non-inferiority GARDEL trial. Lancet Infect Dis. 2014;14:572-80.

27. Margolis DA, Brinson CC, Smith GH, de Vente J, Hagins DP, Eron JJ, et al. Cabotegravir plus rilpivirine, once a day, after induction with cabotegravir plus nucleoside reverse transcriptase inhibitors in antiretroviral-naive adults with HIV-1 infection (LATTE): a randomised, phase 2b, dose-ranging trial. Lancet Infect Dis. 2015;15:1145-55.

28. ClinicalTrials.gov, Regimen Switch to Dolutegravir + Rilpivirine From Current Antiretroviral Regimen in Human Immunodeficiency Virus Type 1 Infected and Virologically Suppressed Adults. Identifier: NCT02429791/NCT02422797 (accessed 21 Apr 2016) 